\title{
Estimation of absolute deformations by changes in distances between the reference points and deformation marks
}

\author{
Olga Tsareva $^{1 *}$, Ivan Dmitriev ${ }^{1}$, and Yuriy Kornilov ${ }^{2}$ \\ ${ }^{1}$ Peter the Great St. Petersburg Polytechnic University, Politechnicheskaya 29, St. Petersburg, \\ 195251, Russian Federation \\ ${ }^{2}$ Saint-Petersburg Mining University, 21st Line 2, St Petersburg, 199106, Russian Federation
}

\begin{abstract}
The article considers the definition of absolute types of deformations, such as transfer and rotation of a building, using linear spatial intersection. In particular, knowing the distances between strong points and deformation marks, the coordinates of the marks located on the building in the initial and current observation cycles are obtained. Knowing the coordinates of the marks, calculate the displacement vectors of the marks for a certain period of time. The definition of absolute deformations is based on the estimation of the projections of the displacement vectors on the coordinate axes, as well as the direction cosines of the vectors with the coordinate axes. The determination of the direction of displacement is shown for the transference deformation. And how to determine the axis, the distance to this axis and the angle of rotation is shown for rotation deformation. We show for joint deformation of the transfer and rotation how divide it into components and then determine the direction of displacement and the axis of rotation. The results can be used in assessing of absolute deformations buildings of monuments of cultural heritage.
\end{abstract}

\section{Introduction}

There are some investigations of various methods of observing deformations and their estimates in articles [1-25]. Deformations can be estimated by knowing the distances between the deformation marks and their changes. The paper [6] dwells on the estimation of the deformation through the distance between deformation marks and their changes. Distances are invariants with respect to the coordinate systems used, which makes it possible to evaluate deformations through a large time interval, regardless of the coordinate system used in the measurements and processing of the results. Deformations are absolute and relative.

Absolute deformations are determined from the support points. Since we work in space and have three coordinates of each of the brands, it is necessary to have at least three strong points, which should not be located on one straight line. Absolute deformations are possible at zero relative. These are included the parallel transfer of the entire object in some direction

\footnotetext{
*Corresponding author: tsareva_os@spbstu.ru
} 
and the rotation with respect to some axis. In this case, the distance between any pair of brand marks in the deformation process does not change, but in general, the distances from brand marks to reference points vary [26-31]. The paper presents studies of a simple case (absolute deformations without relative displacements of object points).

The aim of this article is to develop a method for estimating deformations independent of the coordinate system was posed.

There are some tasks that have been solved:

1. Modeling of transference deformation;

2. Modeling of rotation deformation;

3. Modeling of joint deformation.

\section{Methods}

We investigate the absolute deformations using a linear spatial intersection. In this case, the three points 1,2, 3 are the support points, the lengths of the segments between them are constant. Only the 4-point $\mathrm{P}$ of the tetrahedron changes its position (Fig. 1).

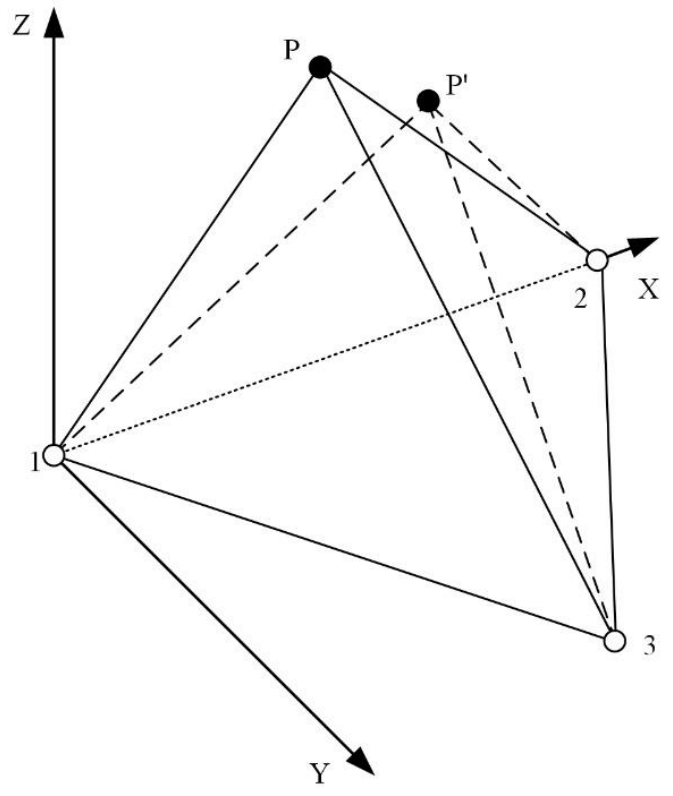

Figure 1. Linear Spatial Resection

Coordinates $X, Y, Z$ can be determined from the solution of the system of three quadratic equations of the form:

$$
L_{i}=\sqrt{\left(X_{i}-X\right)^{2}+\left(Y_{i}-Y\right)^{2}+\left(Z_{i}-Z\right)^{2}}
$$

Where $i=1,2,3$.

But if we choose the coordinate system as shown in the figure 1 (the origin of the coordinate system is aligned with point 1 and the plane $X Y$ coincides with the plane containing the reference marks 1,2 and 3), for which $X_{1}=Y_{1}=Z_{1}=Y_{2}=Z_{2}=Z_{3}=0$, then it is easy to obtain:

$$
X=\left(L_{1}^{2}+X_{2}^{2}-L_{2}^{2}\right) / 2 X_{2}
$$


$Y=\left(Y_{3}^{2}-B+A\right) / 2 Y_{3}$

where $A=L_{1}^{2}-X^{2}, B=L_{3}^{2}-\left(X_{3}-X\right)^{2}$

$$
Y=\left(Y_{3}^{2}-B+A\right) / 2 Y_{3}
$$

It is easy to calculate the coordinate $Z$ from equation (2) for the distance $L_{1}$ :

$$
Z=\sqrt{L_{1}^{2}-X^{2}-Y^{2}}
$$

After calculation the coordinates of both points $P$ and $P^{\prime}$, we can estimate the displacement vector as the coordinate difference:

$$
\begin{aligned}
& u_{X_{P}}=X_{P^{\prime}}^{\prime}-X_{P} \\
& u_{Y_{P}}=Y_{P^{\prime}}^{\prime}-Y_{P} \\
& u_{Z_{P}}=Z_{P^{\prime}}^{\prime}-Z_{P}
\end{aligned}
$$

The initial data are presented in Table 1:

Table 1. Initial data for solving the spatial linear intersection

\begin{tabular}{|l|l|l|l|l|}
\hline No point & $X, \mathrm{~m}$ & $Y, \mathrm{~m}$ & $Z, \mathrm{~m}$ & $L_{i}, \mathrm{~m}$ \\
\hline 1 & 0.000 & 0.000 & 0.000 & 12.800 \\
\hline 2 & 15.867 & 0.000 & 0.000 & 15.439 \\
\hline 3 & 7.865 & 9.711 & 0.000 & 11.008 \\
\hline
\end{tabular}

Using formulas $(2,3)$, we obtain the following coordinates of the point $P$ : $X=5,585 m, Y=5,713 m, Z=10,000 m$.

\section{Results and discussion}

\subsection{Deformation of parallel transfer}

At the first stage, we perform the transfer of point $\mathrm{P}$ parallel to the axes.

\subsubsection{The point's transfer is parallel to the $X$-axis} 2):

With parallel transfer along the $X$-axis of point $P$, we obtain the following data (Table

Table 2. Spatial linear intersection with parallel transfer along the $\mathrm{X}$ axis

\begin{tabular}{|l|l|l|l|l|}
\hline No point & $X, \mathrm{~m}$ & $Y, \mathrm{~m}$ & $Z, \mathrm{~m}$ & $L_{i}, \mathrm{~m}$ \\
\hline 1 & 0.000 & 0.000 & 0.000 & 12.806 \\
\hline 2 & 15.867 & 0.000 & 0.000 & 15.429 \\
\hline 3 & 7.865 & 9.711 & 0.000 & 11.005 \\
\hline$P^{\prime}$ & 5.600 & 5.713 & 10.000 & \\
\hline
\end{tabular}

Define the displacement vector $P P^{\prime}$, its length and the direction cosines (Table 3): 


$$
\begin{aligned}
& u_{X_{P}}=X_{P^{\prime}}^{\prime}-X_{P} \\
& u_{Y_{P}}=Y_{P^{\prime}}^{\prime}-Y_{P} \\
& u_{Z_{P}}=Z_{P^{\prime}}^{\prime}-Z_{P}
\end{aligned}
$$

where $u_{X_{P}}, u_{Y_{P}}, u_{Z_{P}}$ are the coordinates of the displacement vector $P P^{\prime}$;

\begin{tabular}{|c|c|c|c|c|}
\hline \multicolumn{3}{|c|}{ Coordinates of the displacement vector $P P^{\prime}$} & Length of the vector $P P^{\prime}$ & 0.015 \\
\hline 0.015 & 0.000 & 0.000 & & \\
\hline \multicolumn{3}{|c|}{ The direction cosines of a vector $P P^{\prime}$} & & \\
\hline$n_{X_{P}}$ & $n_{Y_{P}}$ & $n_{Z_{P}}$ & & \\
\hline 1 & 0.000 & 0.000 & & \\
\hline
\end{tabular}

$|\bar{u}|=\sqrt{u_{X_{P}}^{2}+u_{Y_{P}}^{2}+u_{Z_{P}}^{2}}$ is its length.

Table 3. The data of the displacement vector $P P^{\prime}$

From Table 3 we see that the cosine of the angle between the vector $P P^{\prime}$ and the $X$ axis is equal 1 . That means the point has changed its position parallel to the $X$ axis. Also the projections of the vector on the coordinate axes $Y$ and $Z$ are 0 . That means the point changed its position perpendicular to the plane $Y Z$. We present the data of tables 2 and 3 in the form:

$$
P=\left|\begin{array}{ccc}
i & j & k \\
u_{X_{P}} & u_{X_{P}} & u_{X_{P}} \\
n_{X_{P}} & n_{X_{P}} & n_{X_{P}}
\end{array}\right|
$$

where $u_{X_{P}}, u_{Y_{P}}, u_{Z_{P}}$ - the coordinates of the displacement vector $P ; n_{X_{P}}, n_{Y_{P}}, n_{Z_{P}}$ - the direction cosines of the displacement vector $P$ of the point with the coordinate axes.

Then for the point $P$ the matrix will look like this:

$$
P=\left|\begin{array}{ccc}
i & j & k \\
0,015 & 0,000 & 0,000 \\
1,000 & 0,000 & 0,000
\end{array}\right|
$$

For a variety of marks which are located on the building, we can form a matrix $A$ of the form:

$$
A=\left|\begin{array}{ccccccc}
i & j & k & & & & \\
u_{X_{P}} & u_{X_{P}} & u_{X_{P}} & & & & \\
n_{X_{P}} & n_{X_{P}} & n_{X_{P}} & & & & \\
& & & \cdots & & & \\
& & & & i & j & k \\
& & & & u_{X_{P}} & u_{X_{P}} & u_{X_{P}} \\
& & & & n_{X_{P}} & n_{X_{P}} & n_{X_{P}}
\end{array}\right|
$$




$$
A=\left|\begin{array}{ccccccc}
i & j & k & & & \\
0,015 & 0,000 & 0,000 & & & \\
1,000 & 0,000 & 0,000 & & & & \\
& & & \ldots & & & \\
& & & & i & j & k \\
& & & & 0,015 & 0,000 & 0,000 \\
& & & & 1,000 & 0,000 & 0,000
\end{array}\right|
$$

All the coordinates of the displacement vectors and the directing cosines will be equal to each other. From the matrix it is easy to determine the value of the displacement of the point, it will be $\Delta=|\bar{u}|=\sqrt{u_{X_{P}}^{2}+u_{Y_{P}}^{2}+u_{Z_{P}}^{2}}=\sqrt{u_{X_{P}}^{2}}=15 \mathrm{MM}$.

\subsubsection{The point's transfer is parallel to the $Y$-axis}

The cosine of the angle between the vector $P P^{\prime}$ and the $Y$ axis will be equal 1 , and the projections of the vector on the $X$ and $Z$ coordinate axes are equal 0 . In the matrix of the form (8), all the coordinates of the displacement vectors will be equal to each other, like the direction cosines. The value of the displacement of the point will be $\Delta=|\bar{u}|=\sqrt{u_{Y_{P}}^{2}}$.

\subsubsection{The point's transfer is parallel to the Z-axis}

The cosine of the angle between the vector $P P^{\prime}$ and the $Z$ axis will be equal 1, also the projections of the vector on the coordinate axes $Y$ and $X$ are equal 0 . Matrix of the form (8) all the coordinates of the displacement vectors will be equal to each other, like the direction cosines. The value of the displacement of the point will be $\Delta=|\bar{u}|=\sqrt{u_{Z_{P}}^{2}}$.

Next, we carry out the transfer of the point $P$ parallel to the coordinate planes $X Y, X Z$, $Z Y$ or perpendicular to the axes $X, Y, Z$.

\subsubsection{The point's transfer is parallel to the $X Y$ plane}

For a variety of marks which are located on the building, we can compose a matrix $A$ :

$$
A=\left|\begin{array}{ccccccc}
i & j & k & & & \\
0,015 & 0,086 & 0,000 & & & & \\
0,172 & 0,985 & 0,000 & & & & \\
& & & \ldots & & & \\
& & & & i & j & k \\
& & & & 0,015 & 0,086 & 0,000 \\
& & & & 0,172 & 0,985 & 0,000
\end{array}\right|
$$

From the matrix we see that the cosine of the angle between the vector $P P^{\prime}$ and the $Z$ axis is equal 0 and the projection of the vector on the coordinate axis $Z$ is equal 0 , which means that the points change their position perpendicular to the $Z$ axis or parallel to the $X Y$ plane. All the coordinates of the displacement vectors will be equal to each other, just like the 
directing cosines. Define the amount of displacement of the point, it will be $\Delta=\sqrt{u_{X_{P}}^{2}+u_{Y_{P}}^{2}}=87 \mathrm{MM}$.

\subsubsection{The point's transfer is parallel to the $Y Z$ plane}

The cosine of the angle between the vector $P P^{\prime}$ and the $X$ axis is equal 0 and the projection of the vector on the $X$ coordinate axis is equal 0 . Also, all the coordinates of the displacement vectors will be equal to each other, like the direction cosines. The value of the displacement of the point is $\Delta=\sqrt{u_{Z_{P}}^{2}+u_{Y_{P}}^{2}}$.

\subsubsection{The point's transfer is parallel to the $X Z$ plane}

The cosine of the angle between the vector $P P^{\prime}$ and the $Y$ axis is equal 0 and the projection of the vector on the coordinate axis $Y$ is equal 0 . Also, all the coordinates of the displacement vectors will be equal to each other, like the direction cosines. The value of the displacement of the point is $\Delta=\sqrt{u_{X_{P}}^{2}+u_{Z_{P}}^{2}}$.

Consider the situation when the point is in the plane with the support points 1, 2, 3 (in case $X Y$ plane) (Table 4$)$.

Table 4. Initial data for solving the spatial linear intersection

\begin{tabular}{|l|l|l|l|l|}
\hline No point & $X, \mathrm{~m}$ & $Y, \mathrm{~m}$ & $Z, \mathrm{~m}$ & $L_{i}, \mathrm{~m}$ \\
\hline 1 & 0.000 & 0.000 & 0.000 & 7.989 \\
\hline 2 & 15.867 & 0.000 & 0.000 & 11.763 \\
\hline 3 & 7.865 & 9.711 & 0.000 & 4.602 \\
\hline
\end{tabular}

Using formulas $(2,3)$, we obtain the following coordinates of the point $P: \mathrm{X}=5.585 \mathrm{~m}$, $\mathrm{Y}=5.713 \mathrm{~m}, \mathrm{Z}=0.000 \mathrm{~m}$.

We research by analogy as in presented case. Carrying the mark parallel to the $X$ and $Y$ axes is easy to identify.

$$
A=\left|\begin{array}{ccccccc}
i & j & k & & & & \\
0,000 & 0,000 & 0,000 & & & & \\
0,000 & 0,000 & 0,000 & & & & \\
& & & \ldots & & & \\
& & & & i & j & k \\
& & & & 0,000 & 0,000 & 0,000 \\
& & & & 0,000 & 0,000 & 0,000
\end{array}\right|
$$

When we carry marks parallel to the $Z$ axis by $10 \mathrm{~mm}$ : 


$$
A=\left|\begin{array}{ccccccc}
i & j & k & & & \\
0,000 & 0,000 & 0,100 & & & & \\
0,000 & 0,000 & 1 & & & & \\
& & & \ldots & & & \\
& & & & i & j & k \\
& & & & 0,000 & 0,000 & 0,100 \\
& & & & 0,000 & 0,000 & 1
\end{array}\right|
$$

If the deformation occurs perpendicular to the plane containing the support and reference marks, the solution will be either completely absent or available depending on the geometry of the tetrahedron and the magnitude of the deformation.

Assume that there was an arbitrary transfer of the object (Fig. 2):

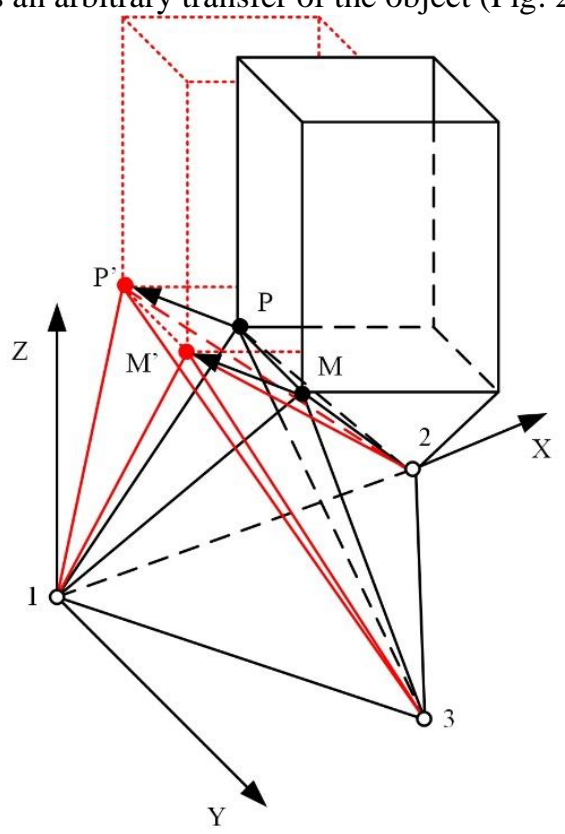

Figure 2. Deformation of the transfer on the example of two deformation marks

Consider two points on the object: $P$ and $M$. The point $P$ has moved to $P^{\prime}$, the point $M$ to $M^{\prime}$.

For all brands located on the object, we form the matrix $A$ :

$$
A=\left|\begin{array}{ccccccc}
i & j & k & & & \\
0,115 & -0,214 & -0,100 & & & & \\
0,438 & -0,814 & -0,831 & & & & \\
& & & \ldots & & & \\
& & & & i & j & k \\
& & & & 0,115 & -0,214 & -0,100 \\
& & & & 0,438 & -0,814 & -0,831
\end{array}\right|
$$

From the matrix (13) we see that the displacement vectors are collinear and equal, and the displacement is arbitrary, since the projections of the vector's coordinates on the axis are 
not equal to 0 and the direction cosines of the displacement vectors have an arbitrary value.

The determined amount of displacement of points is $\Delta=\sqrt{u_{X_{P}}^{2}+u_{Y_{P}}^{2}+u_{Z_{P}}^{2}}=263 \mathrm{MM}$.

\subsection{Deformation of rotation}

\subsubsection{The point's rotation around the $X$-axis}

Perform the rotation of the points $M$ and $D$ (Fig. 3):

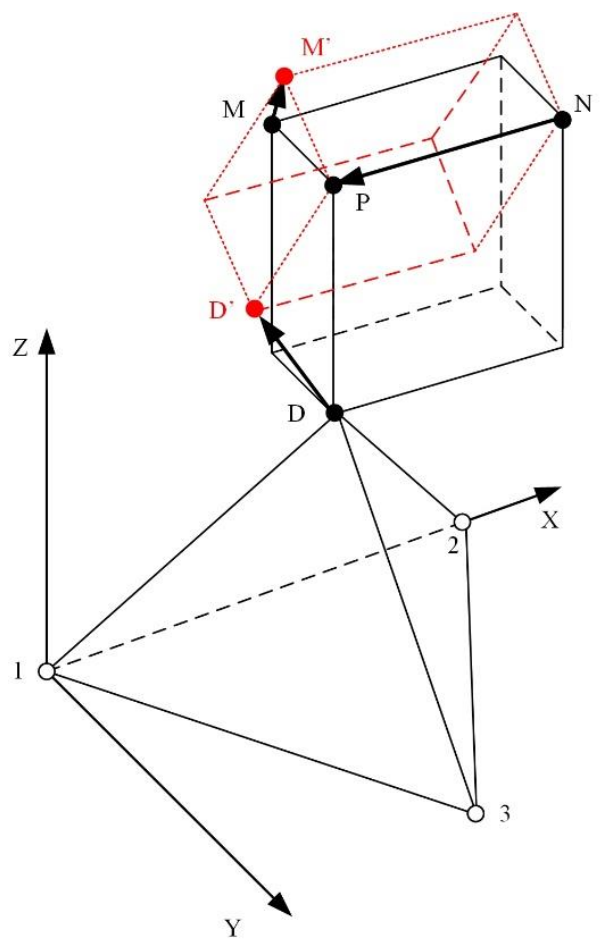

Figure 3. Rotation deformation around the $X$ axis

For a variety of marks, which are located on the building, we can compose the matrix $A$

$$
A=\left|\begin{array}{ccccccc}
i & j & k & & & & \\
0,000 & -0,809 & 3,022 & & & & \\
0,000 & -0,259 & 0,966 & & & & \\
& & & \ldots & & & \\
& & & & i & j & k \\
& & & & 0,000 & 7,500 & 2,011 \\
& & & & 0,000 & 0,966 & -0,259
\end{array}\right|
$$

In contrast to the matrix (10) where the transfer was perpendicular to the $Z$ axis, we see that the matrix components are not equal to each other, which indicates that the deformation of the rotation is not similar as transfer one. In addition, in the case of rotation there will be 
points that have not changed their coordinates (for example, in the figure above the points $N$ and $P$ ), so the displacement vectors of these points will be equal 0 . Then we can define the vector around which the object rotates and find the shortest distance from the displacement vector to the rotation vector [6] by formula (15):

$$
d=\frac{\left|\left(\bar{r}_{2}-\bar{r}_{1}\right) \bar{a}_{1} \bar{a}_{2}\right|}{\sqrt{\left(\bar{a}_{1} \times \bar{a}_{2}\right)^{2}}}
$$

where $\bar{r}_{1}$ is the radius vector of the point located on the axis of rotation (for example, $N$ or $P) ; \bar{r}_{2}$ is the radius vector of the displacement vector point, for example $M, M^{\prime}, D$, $D^{\prime} ; \bar{a}_{1}$ is the directing vector of the first straight line (axis of rotation, in particular $(\overline{N P}$ ); $\bar{a}_{2}$ is the directing vector of the second straight line (for example, the displacement vector $\overline{M M^{\prime}}$ or $\overline{D D^{\prime}}$ ).

We write formula (15) in the form:

$$
d=\frac{\left|\begin{array}{ccc}
X_{2}-X_{1} & Y_{2}-Y_{1} & Z_{2}-Z_{1} \\
l_{1} & m_{1} & n_{1} \\
l_{2} & m_{2} & n_{2}
\end{array}\right|}{\sqrt{\left|\begin{array}{ll}
l_{1} & m_{1} \\
l_{2} & m_{2}
\end{array}\right|^{2}+\left|\begin{array}{ll}
m_{1} & n_{1} \\
m_{2} & n_{2}
\end{array}\right|^{2}+\left|\begin{array}{cc}
n_{1} & l_{1} \\
n_{2} & l_{2}
\end{array}\right|^{2}}}
$$

For example, for points $P$ and $M^{\prime}$ we get:

$$
\begin{aligned}
& \bar{r}_{1}=\left(X_{1}, Y_{1}, Z_{1}\right)=(5,585 ; 5,713 ; 10,000) ; \bar{r}_{2}=\left(X_{2}, Y_{2}, Z_{2}\right)=(5,585 ; 10,948 ; 13,023) \\
& \bar{a}_{1}=\left(l_{1}, m_{1}, n_{1}\right)=(10,000 ; 0 ; 0) ; \bar{a}_{2}=\left(l_{2}, m_{2}, n_{2}\right)=(0 ;-0,809 ; 3,022)
\end{aligned}
$$

The distance to the axis of rotation will be equal to $\mathrm{d}=5.839 \mathrm{~m}$.

Let us calculate the rotation angle $v$ :

$$
v=\operatorname{arctg} \frac{\Delta}{\mathrm{d}}
$$

When $\Delta<<\mathrm{d}$, then $v=\frac{\Delta}{d} \rho$

where $\Delta$ is the magnitude of the displacement (modulus of the displacement vector); $d$ is the shortest distance from the axis of rotation to the point.

$$
v=\operatorname{arctg} \frac{3,129}{5,839}=28^{\circ} 11^{\prime} 09^{\prime \prime}
$$

\subsubsection{The point's rotation around the $Y$-axis and Z-axis}

The cosine of the angle between the displacement vectors and the $Y(Z)$ axis will be equal 0 . The displacement vectors are not collinear and their lengths are not equal to each other. To determine the distance to the rotation axis, we find in the matrix of the form (8) the points whose displacement vectors are zero, calculate the steering vector of rotation and find the distance to the axis of rotation from formula (16). The angle of rotation is calculated according to the formula (17). 


\subsection{Joint deformation of transfer and rotation}

Perform the object transfer parallel to the $X$-axis and rotate around the same axis (Fig. 4):

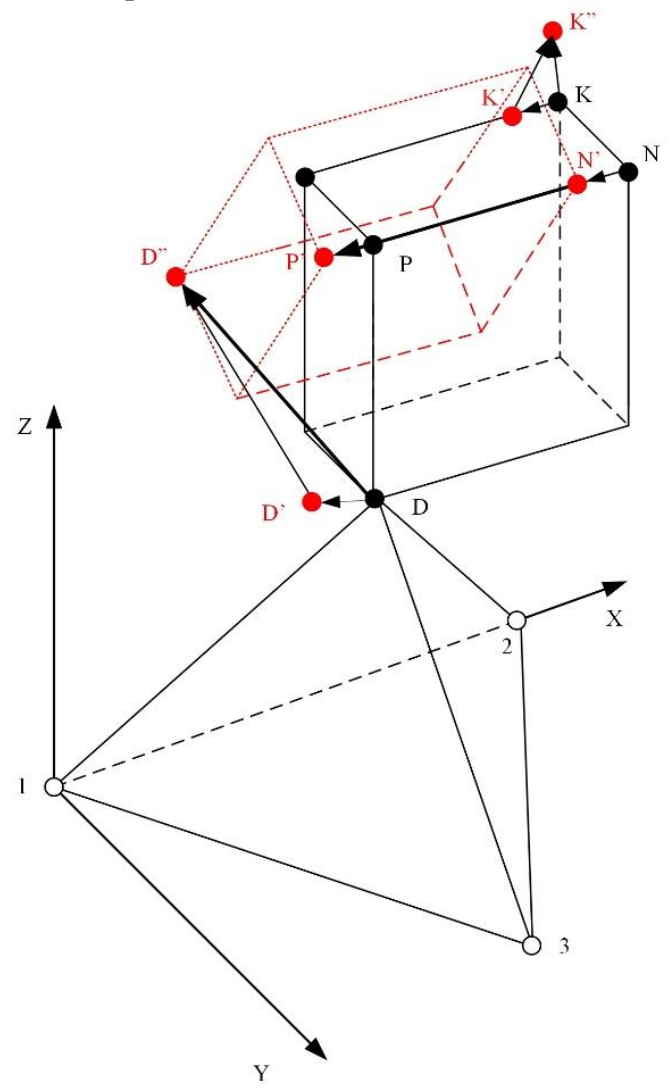

Figure 4. Deformation of the rotation and transfer along to the $X$-axis

From Figure 4 we see that the points $P$ and $N$ change their position only during the transfer (become the points $P^{\prime}$ and $N^{\prime}$ ). The points $D$ and $K$ first go to points $D^{\prime}$ and $K^{\prime}$ , then to $D^{\prime \prime}$ and $K^{\prime \prime}$. Matrix $A$ will be like this:

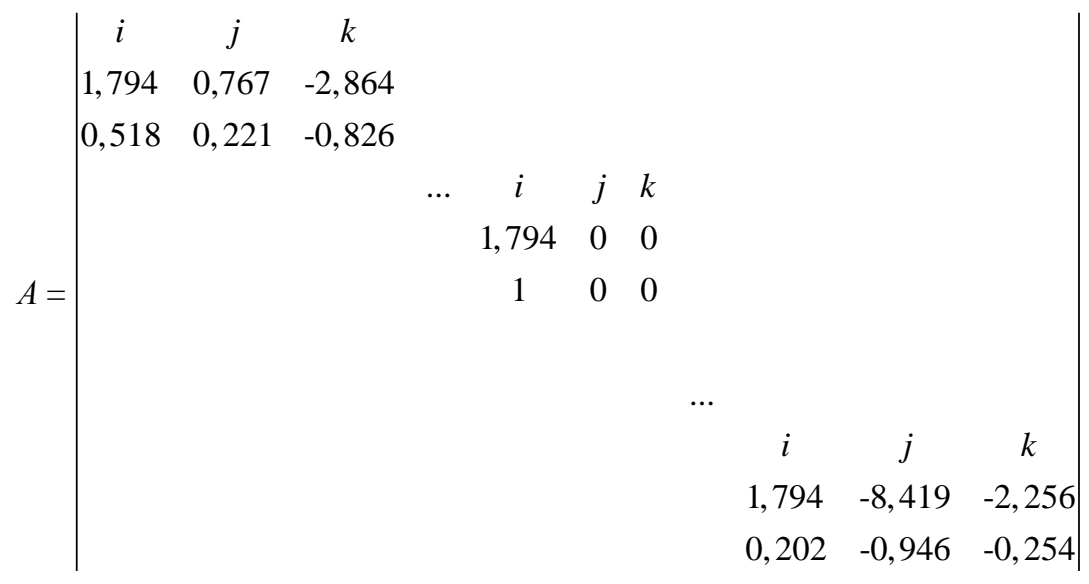


The points $P$ and $N$ obtained only deformation of displacement, and the deformation of rotation for them is equal 0 . PN can be taken as the axis of rotation. This is shown also by the matrix (18), in which the component for these points will be:

$$
P^{\prime}=N^{\prime}=\left|\begin{array}{ccc}
i & j & k \\
1,794 & 0,000 & 0,000 \\
1,000 & 0,000 & 0,000
\end{array}\right|
$$

The matrix shows the transfer along the $X$ axis. Next, subtract the displacement vector from the coordinates of the other displacement vectors. For example, for the point $K$, we get:

$$
\begin{aligned}
K^{\prime \prime} & =\left|\begin{array}{ccc}
i & j & k \\
1,794 & 0,767 & -2,864 \\
0,518 & 0,221 & -0,826
\end{array}\right| \\
K^{\prime} & =\left|\begin{array}{ccc}
i & j & k \\
0 & 0,767 & -2,864 \\
0 & 0,259 & -0,966
\end{array}\right|
\end{aligned}
$$

The resulting matrix (21) shows that there has been a rotation around the $X$ axis. Next, we find the distances from the displacement vectors to the axis of rotation $P N$ according to the formulas 16,17 . The distance to the axis of rotation will be equal to $\mathrm{d}=5.533 \mathrm{~m}$.

The rotation angle $v=\operatorname{arctg} \frac{2,965}{5,533}=28^{\prime \prime} 11^{\prime} 09^{\prime \prime}$

Analogously, one can investigate arbitrary transfers and rotation along one of the axes.

\section{Conclusions}

The completed theoretical modeling allows us to make the following conclusions:

1. To determine the deformations of transfer and rotation, we must consider the direction cosines, which constitute the displacement vector with the coordinate axes and the projection of the vector onto the coordinate axes. We should also consider the mutual position of displacement vectors.

2. In particular, when the building is displaced along one of the coordinate axes, the projections of displacement vectors of deformation marks to other axes are zero, and the directing cosine with the axis with which the displacement occurred is equal to 1 . All the displacement vectors are collinear and equal. If the displacement occurs perpendicular to one of the coordinate axes, the projection of the displacement vector onto the axis perpendicular to which the displacement occurs is equal to 0 and the direction cosine is 0 . All the displacement vectors are collinear and equal to each other. If the deformation occurs perpendicular to the plane containing the support and reference marks, the solution is either completely absent or depends on the displacement and geometry of the tetrahedron.

3. In determining the deformation of the rotation of the displacement vector, deformation matrices are not collinear and are not equal to each other, and the further the displacement vector from the rotation axis, the larger is its length. In particular, when we consider the matrix A rotate, we find points whose displacement vectors, like the direction cosines, turn out to be equal to zero. These points indicate the direction of the axis of rotation. Next, from formula (16), calculate the distance between the rotation axis and any non-zero displacement vector and determine the rotation angle formula (17). 
4. To determine the joint deformation of the transport and rotation in the matrix A, there are points whose matrices of the form (6) are equal. These points have a zero rotation. Estimate the direction and magnitude of the displacement. Next, for the remaining points of the matrix, the displacement component of the found points is subtracted from the displacement vectors of the matrix, a distance is determined by formula (16) between the rotation axis and any nonzero displacement vector and the rotation angle (17) is determined.

\section{References}

1. V.V. Yepin, R.V. Tsvetkov, I.N. Shardakov, Mag. Civ. Eng., 55 (3), 93-94, (2015)

2. A. Benin, A. Konkov, V. Kavkazskiy, A. Novikov, N. Vatin, Proc. Eng., 165, 1483 1489 (2016)

3. B. Kovacic, R. Kamnik, A. Pustovgar, N. Vatin, Proc. Eng., 165, 906-917 (2016)

4. B. Kovacic, R. Kamnik, N. Vatin, A. Ishkov, Proc. Eng., 165, 936-946 (2016)

5. B. Kovacic, R. Kamnik, V. Solovev, N. Vatin, Proc. Eng., 165, 926-935, (2016)

6. V.I. Verkhoturov, A.P. Lashutin, V.V. Petrov, A.M. Sharapov, 28 antennaya konferentsiya Evropeiskogo kosmicheskogo agentstva [28 aerial conference of the European Space Agency], 1-8, (2005)

7. I. Puente, R. Lindenbergh, A. Van Natijne, R. Esposito, R. Schipper, Int. Arch. Photogramm.- ISPRS Archives, 42 (2), 923-929, (2018)

8. L.F. Zhou, P.F. Ma, Y. Xia, C.H. Xie, Int. Arch. Photogramm - ISPRS Archives, 42 (3), 2535-2539, (2018)

9. M. Talich, Y. Bao, W. Guo, G. Wang, W. Gan, M. Zhang, J.S. Shen, E. Kuzina, V. Rimshin, Int. Arch. Photogramm.- ISPRS Archives, 42 (3W4), 499-504, (2018)

10. E. Kuzina, V. Rimshin, Adv. Intell. Syst., 692, 410-416, (2018)

11. Š. Rákay, S. Labant, K. Bartoš, Geodesy and Cartography, 44 (1), 14-21, (2018)

12. M. Talich, IOP Conference Series: Earth and Environmental Science, 95 (3), (2017)

13. H.A. Jaafar, X. Meng, A. Sowter, P. Bryan, Structural Control and Health Monitoring, 24 (11), (2017)

14. X. Zhang, Journal of Physics: Conference Series, 910 (1), (2017).

15. L. Wang, L. Yan, H. Hu, ICCSS 2017 - 2017 International Conference on Information, Cybernetics, and Computational Social Systems, pp. 611-613, (2017)

16. Y.Bao, W.Guo, G.Wang, W.Gan, M.Zhang, J.S.Shen J. Surv. Eng, 144 (1), (2018)

17. M.Wróblewska E3S Web Conf., 36, (2018).

18. J.W. Lovse, W.F. Teskey, G. Lachapelle, M.E. Cannon J. Surv. Eng, 121 (1), 3540, (1995)

19. H.-G. Maas, Photogramm. Eng. Rem. S., 68 (9), 933-940, (2002)

20. V. Janssen, C. Rizos Survey Review, 37 (287), 2-19, (2003)

21. S. Erol, B. Erol, T. Ayan, Int. Arch. Photogramm, Remote Sensing and Spatial Information Sciences - ISPRS Archives, 35, 622-627, (2004).

22. Z.-Y. Hou, D.-J.Yu Wutan Huatan Jisuan Jishu, 26 (3), 256-259, (2004)

23. M. Pieraccini, G. Luzi, D. Mecatti, M. Fratini, F. Parrini, G. Pinelli, C. Atzeni PIERS 2004 - Pr. Electromagn. Res. S., Extended Papers Proceedings, 643-646, (2004) 
24. E. Li, J. Yao Proceedings of SPIE - The International Society for Optical Engineering, 6344 II, (2006)

25. E. Reccia, G. Milani, A. Cecchi, A. Tralli, Constr. Build. Mater. 2014. No. 66 pp. 567-586. (2014)

26. Y.N. V'yunenko, G.A. Volkov, E.A. Khlopkov, Temperature Tech. Phys. 63 (8), 1167-1170 (2018)

27. A.I. Rudskoy, A.A. Bogatov, D.S. Nukhov, A.O. Tolkushkin, Met. Sci. Heat Treat. 60 (1-2), 3-6. (2018)

28. A.M. Bakaeva, A.V. Bakaev, D.A. Terentyev, A.V. Dubinko, E.E. Zhurkin, Thermal J. Surf. Invest. 12 (1), 163-169 (2018)

29. M.A. Skotnikova, V.V. Eliseev, A.A. Moskalets, N.A. Krylov, Int. Rev. Mech. Eng. 11 (5), 350-355 (2017)

30. G.A. Politova, N.Y. Pankratov, P.Y. Vanina, A.V.Filimonov, A.I. Rudskoy, G.S. Burkhanov, A.S. Ilyushin, I.S. Tereshina, J Magn Magn Mater. Article in Press. (2017)

31. O.N. Stepanova, N.N. Vengerova, S.N. Pogodin, Teoriya i Praktika Fizicheskoy Kultury (2015) 